\title{
CALCULATION OF DOMAINS OF DEPENDENCE FOR POLE FIGURES WITH AN ULTRAHYPERBOLIC DIFFERENTIAL EQUATION
}

\author{
T. I. SAVYOLOVA \\ MIFI (Moscow Engineering Physical Institute), Kashirskoe Shosse 31, \\ Moscow 115409, Russia
}

(Received 11 November 1993; in final form 28 September 1994)

$\mathrm{X}$-ray and neutron methods of texture investigation are used to get experimental pole figures $(P F)$ of polcrystalline samples and geological materials (Bunge, 1982). Usually several $P F$ are used, $1-4$ for polycrystalline samples with cubic, hexagonal lattice symmetry (copper, iron, beryllium...), 6-19 for low-symmetry materials of geological samples (quartz, biotite...). Pole figures are the sum of solutions of two ultrahyperbolic equations (Savyolova, 1982). If the solutions of ultrahyperbolic equations are known in some domains we can determine them uniquely in other domains using the Asgeirsson's theorem (Courant, 1962) and their generalizations by ultra-Lorentz transformations. We get the domains of dependence of pole figures and the methods of continuations of solutions of ultrahyperbolic equations.

KEY WORDS: Ultrahyperbolic differential equation, Domain of dependence.

\section{INTRODUCTION}

The orientation distribution function (ODF) in a polycrystalline sample $f(g)$, where $g$ $=\{\alpha, \beta, \gamma\} \in S O(3)$ is the rotation group, $0 \leq \alpha, \gamma<2 \pi, 0 \leq \beta \leq \pi$, are the three Eulerian angles, is determined from experimental pole figures $P_{\overrightarrow{h_{i}}}(\vec{y})$ where $\vec{h}_{i}$ describes the crystal direction in space $R^{3}$ and $\vec{y}$ describes the sample direction in space $R^{3}$.

The ODF $f(g)$ is the solution of the equation (Matthies, 1979)

$P_{\overrightarrow{h_{i}}}(\vec{y})=\hat{P} f(g)=(4 \pi)^{-1} \int_{0}^{2 \pi}\left\{f\left(\{\Phi, \theta, \varphi\}^{-1}\{\eta, \chi, 0\}\right)+f\left(\{\Phi+\pi, \pi-\theta, \varphi\}^{-1}\right.\right.$

$\{\eta, \chi, 0\})\} d \varphi$,

where $\vec{h}_{i}=\{\theta, \Phi\}, \vec{y}=\{\chi, \eta\}, 0 \leq \theta, \chi \leq \pi, 0 \leq \Phi, \eta<2 \pi$ are the spherical coordinates of unit vectors $\vec{h}_{i}, \vec{y}$.

Using the isomorphisms

$$
S O(3) \sim S U(2) /\{ \pm 1\} \sim R P^{3},
$$

where $S O(3)$ is the rotation group, $S U(2)$ is the group of unitary unimodular matrices of second order, $R P^{3}$ is the three-dimensional projective space, $S U(2) /\{ \pm 1\}$ is the factorgroup for subgroup $\{ \pm 1\}, 1$ is the unit of group $S U(2)$, we find (Savyolova, 1982, 1993), that the curves

$$
\pm \vec{h}_{i}=g \vec{y}
$$


can be written as

$$
\vec{x}^{ \pm}=\vec{a}^{ \pm} t+\vec{b}^{ \pm}
$$

with

$$
\begin{aligned}
& \vec{a}^{ \pm}=\left\{-\frac{y_{1} \pm h_{1}}{y_{3} \mp h_{3}},-\frac{y_{2} \mp h_{2}}{y_{3} \mp h_{3}}, 1\right\}, \\
& \vec{b}^{ \pm}=\left\{\frac{y_{2} \pm h_{2}}{y_{3} \mp h_{3}},-\frac{y_{1} \mp h_{1}}{y_{3} \mp h_{3}}, 0\right\},
\end{aligned}
$$

where $\vec{h}_{i}=\left\{h_{1}, h_{2}, h_{3}\right\}, \vec{y}=\left\{y_{1}, y_{2}, y_{3}\right\}$ are the coordinates of unit vectors $\vec{h}_{i}, \vec{y}$ respectively, $h_{3} \neq \pm y_{3}, \vec{x}=\left(x_{1}, x_{2}, x_{3}\right)$ is the point in space $R P^{3},|t|<+\infty$ is a parameter.

Using (2), we get from the equation (1)

$$
\begin{gathered}
P_{\vec{h}_{i}}(\vec{y})=\frac{1}{\left|h_{3}-y_{3}\right|} F^{+}\left(\vec{a}^{+}, \vec{b}^{+}\right)+\frac{1}{\left|y_{3}+h_{3}\right|} F^{-}\left(\vec{a}^{-}, \vec{b}-\right), \\
F^{ \pm}\left(\vec{a}^{ \pm}, \vec{b} \pm\right)=\int_{-\infty}^{+\infty} \frac{8 f\left(\vec{a}^{ \pm} t+\vec{b}^{ \pm}\right) d t}{A^{ \pm} t^{2}+2 B^{ \pm} t+C^{ \pm}}
\end{gathered}
$$

where

$$
\begin{gathered}
A^{ \pm}=\left(a_{1}^{ \pm}\right)^{2}+\left(a_{2}^{ \pm}\right)^{2}+1, \quad C^{ \pm}=\left(b_{1}^{ \pm}\right)^{2}+\left(b_{2}^{ \pm}\right)^{2}+1, \\
B^{ \pm}=a_{1}^{ \pm} b_{1}^{ \pm}+a_{2}^{ \pm} b_{2}^{ \pm} .
\end{gathered}
$$

From the equation (3) we get the ultrahyperbolic differential equations for pole figures

$$
\frac{\partial^{2} F^{ \pm}}{\partial a_{1}^{ \pm} \partial b_{2}^{ \pm}}=\frac{\partial^{2} F^{ \pm}}{\partial a_{2}^{ \pm} \partial b_{1}^{ \pm}}
$$

where

$$
\begin{gathered}
a_{1}^{ \pm}=-\frac{y_{1} \pm h_{1}}{y_{3} \mp h_{3}}, \quad a_{2}^{ \pm}=-\frac{y_{2} \mp h_{2}}{y_{3} \mp h_{3}}, \\
b_{1}^{ \pm}=\frac{y_{2} \pm h_{2}}{y_{3} \mp h_{3}}, \quad b_{2}^{ \pm}=-\frac{y_{1} \mp h_{1}}{y_{3} \mp h_{3}} .
\end{gathered}
$$

We have $\vec{x}=\left(x_{1}, x_{2}, x_{3}\right) \in R P^{3}$ through the Eulerian angles $g=\{\alpha, \beta, \gamma\} \in S O(3)$

$$
\begin{gathered}
x_{1}=\left(\operatorname{ctan} \frac{\beta}{2}\right)\left(\cos \frac{\alpha+\gamma}{2}\right)\left(\cos \frac{\alpha-\gamma}{2}\right)^{-1}, \\
x_{2}=\left(\operatorname{ctan} \frac{\beta}{2}\right)\left(\sin \frac{\alpha+\gamma}{2}\right)\left(\cos \frac{\alpha-\gamma}{2}\right)^{-1}, \\
x_{3}=-\tan \frac{\alpha-\gamma}{2} .
\end{gathered}
$$

The Jacobian of transformation (5) is

$$
\frac{\partial(\alpha, \beta, \gamma)}{\partial\left(x_{1}, x_{2}, x_{3}\right)}=4\left(1+x_{3}^{2}\right)^{-\frac{1}{2}}\left(x_{1}^{2}+x_{2}^{2}\right)^{-\frac{1}{2}}\left(1+x_{1}^{2}+x_{2}^{2}+x_{3}^{2}\right)^{-1}
$$


Remark. Let us have pole figures in spherical coorinates

$$
P_{\vec{h}_{i}}(\vec{y}) \equiv F(\Phi, \theta, \eta, \chi)=\sum_{l=0}^{\infty} \sum_{m, n=-1}^{l} C_{m n}^{l} Y_{m}^{l}(\vec{y})\left[Y_{n}^{l}\left(\vec{h}_{i}\right)\right]^{*},
$$

where $\vec{h}_{i}=\{\theta, \Phi\}, \vec{y}=\{\chi, \eta\}, 0 \leq \theta, \chi \leq \pi, 0 \leq \Phi, \eta<2 \pi$ are the spherical coordinates of the unit vectors $h_{i}, \vec{y}$,

$$
\begin{aligned}
& Y_{m}^{l}(\vec{y})=(2 \pi)^{-\frac{1}{2}} \exp \{-i m \eta\} P_{m}^{l}(\cos \chi), \\
& {\left[Y_{n}^{l}\left(\overrightarrow{h_{i}}\right)\right]^{*}=(2 \pi)^{-\frac{1}{2}} \exp \{i n \Phi\} P_{n}^{l}(\cos \theta),}
\end{aligned}
$$

$P_{m}^{l}(\cos \chi)$ are generalized Legendre polynomials.

Equation (4) corresponds to the differential equation for $P F$ (6) in spherical coordinates (Savyolova, 1988)

$$
\left(1-z^{2}\right) \frac{\partial^{2} F}{\partial z^{2}}-2 z \frac{\partial F}{\partial z}+\frac{1}{1-z^{2}} \frac{\partial^{2} F}{\partial \eta^{2}}=\left(1-w^{2}\right) \frac{\partial^{2} F}{\partial w^{2}}-2 w \frac{\partial F}{\partial w}+\frac{1}{1-w^{2}} \frac{\partial^{2} F}{\partial \Phi^{2}},
$$

where $z=\cos \chi, w=\cos \theta$.

\section{Ultrahyperbolic equations}

Let

$$
\begin{array}{ll}
x_{1}^{ \pm}=-\frac{2 y_{1}}{y_{3} \mp h_{3}}, & x_{2}^{ \pm}=-\frac{2 y_{2}}{y_{3} \mp h_{3}}, \\
z_{1}^{ \pm}= \pm \frac{2 h_{2}}{y_{3} \mp h_{3}}, & z_{2}^{ \pm}=\mp \frac{2 h_{1}}{y_{3} \mp h_{3}},
\end{array}
$$

then we get from equations (4)

$$
\frac{\partial^{2} F^{ \pm}}{\partial\left(x_{1}^{ \pm}\right)^{2}}+\frac{\partial^{2} F^{ \pm}}{\partial\left(x_{2}^{ \pm}\right)^{2}}=\frac{\partial^{2} F^{ \pm}}{\partial\left(z_{1}^{ \pm}\right)^{2}}+\frac{\partial^{2} F^{ \pm}}{\partial\left(z_{2}^{ \pm}\right)^{2}}
$$

Consider the problem of solution continuation of ultrahyperbolic differential equations (7). We drop the supercript " \pm " in equations (7).

Using the Asgeirsson's theorem we have the following result (Ivanova, Savyolova, 1993) : if the function $F(\vec{x}, \vec{z})$ is solution of equation (7), even in the variable $z_{2}-z_{2}^{0}=t$ and is known in the domain

$$
\left\{\sum_{i=1}^{2}\left(x_{i}-x_{i}^{0}\right)^{2} \leq a^{2}, \quad \sum_{i=1}^{2}\left(z_{i}-z_{i}^{0}\right)^{2} \leq \varepsilon^{2}\right\}
$$

then it is determined uniquely in the cone

$$
\left\{\sum_{i=1}^{2}\left(x_{i}-x_{i}{ }^{0}\right)^{2}\right\}^{\frac{1}{2}}+\left\{\sum_{i=1}^{2}\left(z_{i}-z_{i}^{0}\right)^{2}\right\}^{\frac{1}{2}} \leq a \quad(a>\varepsilon) .
$$

This result can be generalized for many other domains by ultra-Lorentz transformations

$$
\begin{aligned}
& x_{1}^{\prime}=\left(x_{1}+\beta z_{1}\right)\left(1-\beta^{2}\right)^{-\frac{1}{2}}, \\
& x_{2}^{\prime}=\left(x_{2}+\alpha z_{2}\right)\left(1-\alpha^{2}\right)^{-\frac{1}{2}}, \\
& z_{1}^{\prime}=\left(\beta x_{1}+z_{1}\right)\left(1-\beta^{2}\right)^{-\frac{1}{2}}, \\
& z_{2}^{\prime}=\left(\alpha x_{2}+z_{2}\right)\left(1-\alpha^{2}\right)^{-\frac{1}{2}},
\end{aligned}
$$

with different parameters $|\alpha|,|\beta|<1$. 
Let $x_{1}^{0}=x_{2}^{0}=z_{1}^{0}=z_{2}^{0}=0$ in (8), (9). Consider the method of continuation of solution of ultrahyperbolic equation (7). Let

$$
\begin{gathered}
r=\sqrt{x_{1}^{2}+x_{2}^{2}}, \quad \tan \varphi=\frac{x^{2}}{x_{1}}, \quad \rho=\sqrt{z_{1}^{2}+z_{2}^{2}}, \quad \tan \emptyset=\frac{z^{2}}{z_{1}}, \\
F(r, \varphi, \rho, \emptyset)=F(\vec{x}(r, \varphi), \vec{z}(\rho, \emptyset))_{r=a}=0 .
\end{gathered}
$$

From equation (7) in polar coordinates

$$
\frac{1}{r} \frac{\partial}{\partial r}\left(r \frac{\partial F}{\partial r}\right)+\frac{1}{r^{2}} \frac{\partial^{2} F}{\partial \varphi^{2}}=\frac{1}{\rho} \frac{\partial}{\partial \rho}\left(\rho \frac{\partial F}{\partial \rho}\right)+\frac{1}{\rho^{2}} \frac{\partial^{2} F}{\partial \phi^{2}},
$$

we get the solutions

$$
J_{n}\left(\lambda \frac{r}{a}\right) J_{m}\left(\lambda \frac{\rho}{a}\right) e^{i(n \varphi+m \text { ø) }}
$$

because $J_{n}\left(\lambda \frac{r}{a}\right) e^{i(n \varphi)}$ are the eigenfunctions of Laplace operator in polar coordinates $(r, \varphi)$ corresponding to the eigenvalue $-\left(\frac{\lambda}{a}\right)^{2}$ (Vilenkin, 1965).

Thus we have

$$
F(r, \varphi, \rho, \emptyset)=\sum_{m, n=-\infty}^{+\infty} \sum_{k=0}^{+\infty} A_{m n k} J_{n}\left(\lambda_{n k} \frac{r}{a}\right) J_{m}\left(\lambda_{n k} \frac{\rho}{a}\right) e^{i(n \varphi+m \emptyset)}
$$

where $\lambda_{n k}$ are the roots of equations for Bessel's functions $J_{n}\left(\lambda_{n k}\right)=0$. The coefficients $A_{m n k}$ can be calculated by the formulae

$$
\begin{gathered}
A_{m n k}=\lim _{\varepsilon \rightarrow 0} \frac{\int_{0}^{\varepsilon} F_{m n k}(\rho) d \rho}{2 \pi^{2} a^{2} J_{n+1}^{2}\left(\lambda_{n k}\right) \int_{0}^{\varepsilon} J_{m}\left(\lambda_{n k} \frac{\rho}{a}\right) d \rho}, \\
F_{m n k}(\rho)=\int_{0}^{a} \int_{0}^{2 \pi} \int_{0}^{2 \pi} F(r, \varphi, \rho, \emptyset) r J_{n}\left(\lambda_{n k} \frac{r}{a}\right) e^{-i(n \varphi+m \emptyset)} d r d \varphi d \phi .
\end{gathered}
$$

Using the theorem of sum of Bessel's functions (Korn, 1973), we have

$$
J_{n+m}\left(\lambda \frac{r}{a}\right) J_{m}\left(\lambda \frac{\rho}{a}\right)=\frac{2}{\pi} \int_{|r+\rho|}^{r+\rho} \frac{R J_{n}\left(\lambda \frac{R}{a}\right) e^{-i m(\varphi+\phi)+i n w} d R}{\sqrt{4 r^{2} \rho^{2}-\left(R^{2}-r^{2}-\rho^{2}\right)^{2}}} \operatorname{sgnsin}(\varphi+\phi),
$$

where

$$
R^{2}=r^{2}+\rho^{2}-2 r \rho \cos (\varphi+\varnothing), \quad e^{2 i w}=\frac{r-\rho e^{-i(\varphi+\phi)}}{r-\rho e^{i(\varphi+\phi)}} .
$$

Thus if the function $F(r, \varphi, \rho, \varnothing)$ is known in domain $0 \leq r \leq a, 0 \leq \rho \leq \varepsilon$, then we can calculate the series (10) with the coefficients (11). But the right-hand part of expression (10) is known in the domain $0 \leq r+\rho \leq a$ because in formula (12) the variables $r$ and $\rho$ are equivalent (interchangeable).

Consequently we have

Theorem 1. If the function $F(\vec{x}, \vec{z})$ is known in the domain (8), then it is determined in the cone (9) by formulae (10), (11).

There are some difficulties with the choice of boundary condition for the function $F(r, \varphi, \rho, \emptyset)$ when $r=a$. Apparently the boundary condition $\left.\frac{\partial F}{\partial r}\right|_{r=a}=0$ is less restrictive. In that case $\lambda_{n k}$ in the formula (10) are the roots of equation $J_{n}^{\prime}\left(\lambda_{n k}\right)=0, k=1,2, \ldots$. 
If we have $a=\infty$, then we can determine the function $F(r, \varphi, \rho, \varnothing)$ for $0 \leq$ $r+\rho<+\infty$ by using the Fourier-Bessel transform

$$
F(r, \varphi, \rho, \emptyset)=\sum_{m, n=-\infty}^{+\infty}\left(\int_{0}^{+\infty} A_{m n}(\lambda) J_{n}(\lambda r) J_{m}(\lambda \rho) \lambda d \lambda\right) e^{i(n \varphi+m \varnothing)},
$$

where

$$
A_{m n}(\lambda) J_{m}(\lambda \rho)=(2 \pi)^{2} \int_{0}^{2 \pi} \int_{0}^{2 \pi} \int_{0}^{+\infty} r F(r, \varphi, \rho, \varnothing) e^{-i(n \varphi+m \varnothing)} J_{n}(\lambda r) d r d \varphi d \phi .
$$

\section{The domains of dependence of pole figures}

Considers the applications of the given results to the problem of calculating domains of dependence of pole figures for polycrystalline samples with different lattice symmetries. We have the variables

$$
\vec{x}^{ \pm}=\left(x_{1}^{ \pm}, x_{2}^{ \pm}\right), \quad \vec{z}^{ \pm}=\left(z_{1}^{ \pm}, z_{2}^{ \pm}\right)
$$

in spherical coordinates of the unit vectors

$$
\begin{gathered}
\vec{h}_{i}=\{\theta, \Phi\}, \quad y=\{\chi, \eta), \quad 0 \leq \theta, \quad \chi \leq \pi, \quad 0 \leq \Phi, \quad \eta<2 \pi, \\
x_{1}^{ \pm}=-\frac{2 \sin \chi \cos \eta}{\cos \chi \mp \cos \theta}, \quad x_{2}^{ \pm}=-\frac{2 \sin \chi \sin \eta}{\cos \chi \mp \cos \theta}, \\
z_{1}^{ \pm}= \pm \frac{2 \sin \theta \sin \Phi}{\cos \chi \mp \cos \theta}, \quad z_{2}^{ \pm}=\mp \frac{2 \sin \theta \cos \Phi}{\cos \chi \mp \cos \theta}, \quad \cos \chi \neq \pm \cos \theta .
\end{gathered}
$$

For polar coordinates we get

$$
r^{ \pm}=\frac{2 \sin \chi}{|\cos \chi \mp \cos \theta|}, \quad \rho^{ \pm}=\frac{2 \sin \theta}{|\cos \chi \mp \cos \theta|}, \quad \varphi=\eta, \quad \emptyset=\Phi .
$$

We have the following implication from (3), (8), (9).

If the functions $F^{ \pm}$are known in the domains

$$
D^{ \pm}=\left\{\sin \chi \leq \frac{a}{2}|\cos \chi \mp \cos \theta|, \sin \theta \leq \frac{\varepsilon}{2}|\cos \chi \mp \cos \theta|\right\},
$$

then they are determined uniquely in the domains

$$
G^{ \pm}=\left\{(\sin \chi+\sin \theta) \leq \frac{\max (a, \varepsilon)}{2}|\cos \chi \mp \cos \theta|\right\}
$$

The application of the results for continuation of solutions of ultrahyperbolic equations for pole figures is in (Ivanova, Savyolova, 1993). We cannot distinquish every component $F^{+}$and $F^{-}$for all pole figures except one $P F$ with $h_{0}=\left\{\theta_{0}, \Phi_{0}\right\}$, $\theta_{0}=\frac{\pi}{2}$. The equality $F^{+}=F^{-}$holds for this $P F$.

From $P F$ with $h=\left(\theta_{0}, \Phi_{0}\right), \theta_{0}=\frac{\pi}{2}$ the function $F(r, \varphi, \rho, \varnothing)$ is known for $\rho=\sqrt{r^{2}+4}$, $\emptyset=\emptyset_{0}=\Phi_{0}, 0 \leq r<+\infty, 0 \leq \varphi<2 \pi$.

We can calculate

$$
F_{n}\left(r, \rho, \emptyset_{0}\right)=\int_{0}^{2 \pi} F(r, \varphi, \rho, \emptyset) e^{-i n \varphi} d \varphi,
$$

when $\rho=\sqrt{r^{2}+4}, n=0, \pm 1, \ldots$. 
The function $F_{n}\left(r, \rho, \emptyset_{0}\right)$ with $\rho=\sqrt{r^{2}+4}$ can be defined with the formula (10) by the series representation

$$
F_{n}\left(r, \rho, \emptyset_{0}\right)=\sum_{m=-\infty}^{+\infty} \sum_{k=0}^{\infty} A_{m n k} J_{n}\left(\lambda_{n k} \frac{r}{a}\right) J_{m}\left(\lambda_{n k} \frac{\rho}{a}\right) e^{i m \phi_{0}}(2 \pi)
$$

Denote the integral for the function $F_{n}\left(r, \sqrt{r^{2}+4}, \emptyset_{0}\right)$ by

$$
F_{n p s}=\int_{0}^{a} F_{n}\left(r, \sqrt{r^{2}+4}, \emptyset_{0}\right) r J_{s}\left(\lambda_{s p} \frac{r}{a}\right) d r\left[\frac{a^{2}}{2} J_{s+1}^{2}\left(\lambda_{s p}\right)\right] .
$$

Using the formulas (16), (17) we get the system of algebraic equations for the unknown coefficients $A_{m n k}$

$$
F_{n p s}=\sum_{m=-\infty}^{+\infty} \sum_{k=0}^{\infty} A_{m n k} B_{m n k p s}
$$

where

$$
B_{m n k p s}=(2 \pi) e^{i m \phi_{0}} \int_{0}^{a} r J_{s}\left(\lambda_{s p} \frac{r}{a}\right) J_{n}\left(\lambda_{n k} \frac{r}{a}\right) J_{m}\left(\lambda_{n k} \frac{\sqrt{r^{2}+4}}{a}\right) d r,
$$

$m, n, s=0, \pm 1, \ldots, k, p=0,1, \ldots$

Then all $P F$ can be determined in the domains $2 \leq r^{ \pm}+\rho^{ \pm} \leq a(a>2)$ by formulas (3), (10) with the calculated coefficients $A_{m n k}$. The system has the unique solution when the function $F$ does not depend on the variable $\varnothing$.

Let us consider the application of these results to materials with every kind of lattice symmetry. If we know $P F$ with $\vec{h}_{o}=\left\{\frac{\pi}{2}, \Phi_{0}\right\}$, the function $F(r, \varphi, \rho)$ can be calculated in the domain

$$
D=\{r+\rho \geq 2\} .
$$

Then every $P F$ with $\vec{h}_{i}=\left\{\theta_{i}, \Phi_{i}\right\}, 0<\theta_{i}<\frac{\pi}{2}$, can be determinated by the formula (3) in the domain

$$
G=\left\{\frac{\pi}{2}-\theta_{i} \leq \chi \leq \frac{\pi}{2}+\theta_{i}\right\}
$$

where $G=G^{+} \cap G^{-}$,

$$
G^{ \pm}=\left\{r^{ \pm}+\rho^{ \pm} \geq 2\right\}=\left\{\frac{\sin \chi+\sin \theta_{i}}{\left|\cos \chi \mp \cos \theta_{i}\right|} \geq 1\right\} \quad \text { (see appendix). }
$$

If $P F$ with $\vec{h}_{o}=\left\{\frac{\pi}{2}, \Phi_{0}\right\}$ is known partially in the domain

$$
D=\{2 \leq r+\rho \leq a\}=\left\{0 \leq \chi \leq \chi_{1}, \pi-\chi_{1} \leq \chi \leq \pi\right\},
$$

where $2<a<+\infty$

$$
\chi_{1}=\cos ^{-1}\left(\frac{4 a}{a^{2}+4}\right)
$$

then every $P F$ with $\vec{h}_{i}=\left\{\theta_{i}, \Phi_{i}\right\}, 0<\theta_{i}<\frac{\pi}{2}$, can be calculated in the domain

$$
G=G^{+} \cap G^{-}, \quad G^{ \pm}=\left\{2 \leq r^{ \pm}+\rho^{ \pm} \leq a\right\}=\left\{1 \leq \frac{\sin \chi+\sin \theta_{i}}{\left|\cos \chi \mp \cos \theta_{i}\right|} \leq \frac{a}{2}\right\} .
$$


Two cases are possible. Let $2 \leq a \leq 2(2+\sqrt{3})$, then $0<\theta^{*} \leq \frac{\pi}{6}$, where

$$
\theta^{*}=\tan ^{-1}\left(\frac{a}{2}\right)-\frac{\pi}{4} \text {. }
$$

In this case we have 4 possibilities (see appendix):

1a) if $\frac{\pi}{2}-\theta^{*}<\theta_{i}<\frac{\pi}{2}$, then

$$
G=\left\{\frac{\pi}{2}-\theta_{i}<\chi<-\frac{\pi}{2}+\theta_{i}+2 \theta^{*}, \quad \frac{3 \pi}{2}-2 \theta^{*}-\theta_{i}<\chi<\frac{\pi}{2}+\theta_{i}\right\} ;
$$

1b) if $2 \theta^{*}<\theta_{i}<\frac{\pi}{2}-\theta^{*}$, then

$$
G=\varnothing
$$

1c) if $\theta^{*}<\theta_{i}<2 \theta^{*}$, then

$$
G=\left\{\frac{3 \pi}{2}-2 \theta^{*}+\theta_{i}<\chi<\frac{\pi}{2}+2 \theta^{*}-\theta_{i}\right\}
$$

1d) if $0<\theta_{i}<\theta^{*}$, then

$$
G=\left\{\frac{\pi}{2}-\theta_{i}<\chi<\frac{\pi}{2}+\theta_{i}\right\}
$$

In Figures $1,1 a-1 d$ the domain $G$ with $2 \leq a<+\infty$ is shown as a black portion of the entire domain $G$ with $a=\infty$ that can be determined from $P F$ with $\vec{h}_{o}=\left\{\frac{\pi}{2}, \Phi_{0}\right\}$. The difference between them, corresponding to the domain

$$
\left\{r^{ \pm}+\rho^{ \pm} \geq a\right\}
$$

is hatched in Figures $1,1 a-1 d$. Four variants $1 a-1 d$ for the domain $G$ are given in Figures $1 a-1 d$ respectively.

The domain $\left\{r^{ \pm}+\rho^{ \pm} \geq 2\right\}$ cannot be determined from $P F$ with $\vec{h}_{o}=\left\{\frac{\pi}{2}, \Phi_{0}\right\}$ (blank part of $P F$ in Figures $1 a-1 d)$.

In the second case we have $a>2(2+\sqrt{3}), \theta^{*}>\frac{\pi}{6}$ (see appendix). Let

$$
\theta^{* *}=\frac{3 \pi}{4}-\tan ^{-1}\left(\frac{a}{2}\right)=\frac{\pi}{2}-\theta^{*} \text {. }
$$

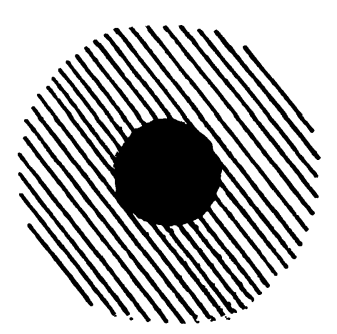

Figure 1 The domain $D$ with $2<a<2(2+\sqrt{3})$ (black) and the other part of $P F$ with $\vec{h}_{o}=\left\{\frac{\pi}{2}, \Phi_{0}\right\}$ (hatched). 


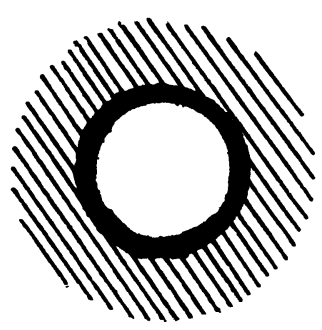

Figure 1a The domain $G$ on $P F$ with $\vec{h}_{i}=$ $\left\{\theta_{i}, \Phi_{i}\right\}$, when $\frac{\pi}{2}-\theta^{*}<\theta_{i}<\frac{\pi}{2}$.

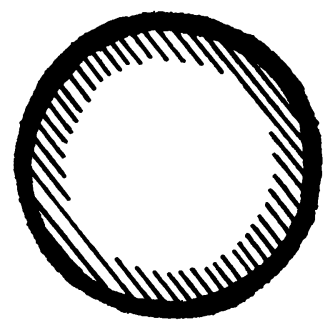

Figure 1c The domain $G$ on $P F$ with $\theta^{*}<\theta_{i}$ $<2 \theta^{*}$.

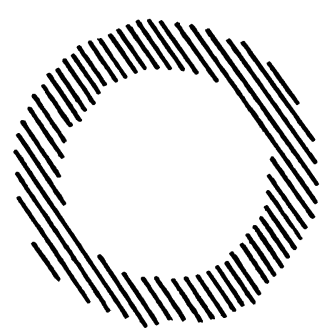

Figure $1 \mathrm{~b}$ The domain $G$ on $P F$ with $2 \theta^{*}<$ $\theta_{i}<\frac{\pi}{2}-\theta^{*}$.

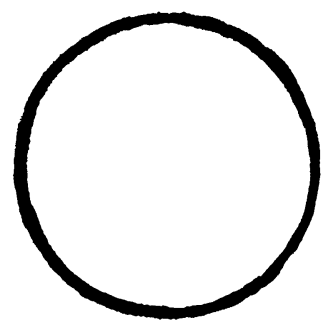

Figure 1d The domain $G$ on $P F$ with $0<\theta_{i}$ $<\theta^{*}$.

Four variants are possible:

$2 a)$ if $2 \theta^{*}<\theta_{i}<\frac{\pi}{2}$, then

$$
G=\left\{\frac{\pi}{2}-\theta_{i}<\chi<-\frac{\pi}{2}+2 \theta^{*}+\theta_{i}, \frac{3 \pi}{2}-2 \theta^{*}-\theta_{i}<\chi<\frac{\pi}{2}+\theta_{i}\right\} ;
$$

2b) if $\theta^{* *}<\theta_{i}<2 \theta^{*}$, then

$$
G= \begin{cases}\frac{\pi}{2}-\theta_{i} & <\chi<-\frac{\pi}{2}+2 \theta^{*}+\theta_{i} \\ \frac{3 \pi}{2}-2 \theta^{*}+\theta_{i} & <\chi<2 \theta^{*}-\frac{\pi}{2}-\theta_{i} \\ \frac{3 \pi}{2}-2 \theta^{*}-\theta_{i} & <\chi<\frac{\pi}{2}+\theta_{i}\end{cases}
$$

2c) if $\theta^{*}<\theta_{i}<\theta^{* *}$, then

$$
G=\left\{\frac{3 \pi}{2}-2 \theta^{*}+\theta_{i}<\chi<2 \theta^{*}-\frac{\pi}{2}-\theta_{i}\right\}
$$

2d) if $0<\theta_{i}<\theta^{*}$, then

$$
G=\left\{\frac{\pi}{2}-\theta_{i}<\chi<\frac{\pi}{2}+\theta_{i}\right\}
$$




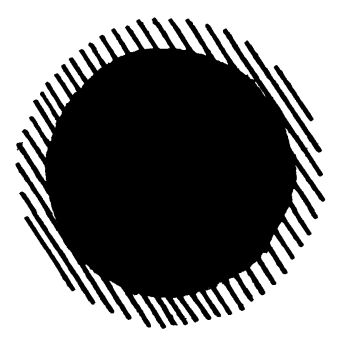

Figure 2 The domain $D$ with $a>2(2+\sqrt{3})$ (black) and the other part of $P F$ with $\vec{h}_{o}=\left\{\frac{\pi}{2}, \Phi_{0}\right\}$ (hatched).

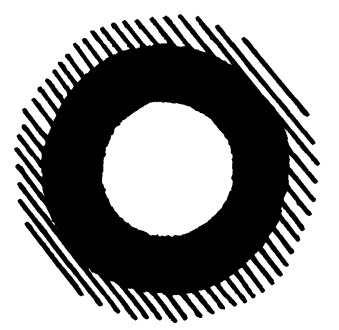

Figure $2 \mathrm{a}$ The domain $G$ for $2 \theta^{*}<\theta_{i}<\frac{\pi}{2}$.

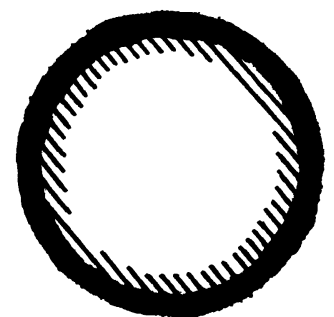

Figure 2c The domain $G$ for $\theta^{*}<\theta_{i}<\theta^{* *}$.

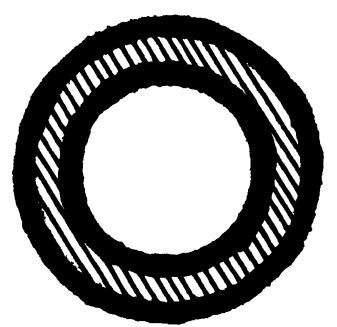

Figure 2b The domain $G$ for $\theta^{* *}<\theta_{i}<2 \theta^{*}$.

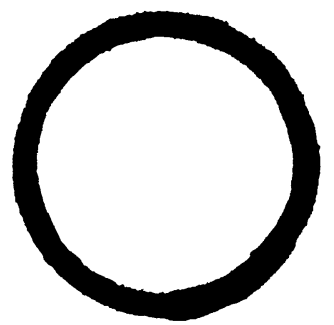

Figure 2d The domain $G$ for $0<\theta_{i}<\theta^{*}$.

In Figures $2,2 a-2 d$ the domain $G$ with $2(2+\sqrt{3}) \leq a<+\infty$ and the domain with

$$
\left\{r^{ \pm}+\rho^{ \pm} \geq a\right\}
$$

are marked off like in the first case. By contrast to the Figures $1,1 a-1 d$ for every $P F$ with $0<\theta_{i}<\frac{\pi}{2}$ the domain $G$ is not equal to zero (see figure $1 b$ ), because in the second case the parameter $a$ is greater than in the first case. The domain $G$ has the form of two rings in Figure $2 b$. For every $a \geq 2$ for $P F$ with $\vec{h}_{i}=\left\{\theta_{i}, \Phi_{i}\right\}, \theta_{i}=0$, we have $G=\emptyset$. 
If in formula (10) we have no variables $\varphi, \varnothing \in[0,2 \pi]$ then we can get every pole figure from one $P F$ with $\vec{h}_{0}=\left\{\theta_{0}, \Phi_{0}\right\}, \theta_{0}=\frac{\pi}{2}$ from its analytical formula by replacing

with

$$
r=\frac{2 \sin \chi}{|\cos \chi|}, \quad \rho=\frac{2}{|\cos \chi|}
$$

$$
r^{ \pm}=\frac{2 \sin \chi}{|\cos \chi \mp \cos \theta|}, \quad \rho^{ \pm}=\frac{2 \sin \theta}{|\cos \chi \mp \cos \theta|}
$$

respectively.

For example, for approximation of $P F\{10 \overline{10}\}$ for a simple axial texture of beryllium in Figure $3 a$ we have

$$
P_{\{10 \overline{0}\}}=\frac{1}{\varepsilon^{2}} \exp \left\{\frac{-\beta^{2}}{4 \varepsilon^{2}}\right\}
$$

with $\varepsilon=0.62$

$$
\cos \beta=\frac{16-\left(r^{2}-\rho^{2}\right)^{2}+16 r \rho}{16+\left(r^{2}-\rho^{2}\right)^{2}+8\left(r^{2}-\rho^{2}\right)}, \quad r=\frac{2 \sin \chi}{|\cos \chi|}, \quad \rho=\frac{2}{|\cos \chi|} .
$$

For every $P F$ with $\vec{h}=\{\theta, \Phi\}, 0 \leq \theta \leq \pi, 0 \leq \Phi<2 \pi$ we get the formula

$$
\begin{gathered}
P_{\vec{h}}\left(r^{ \pm}, \rho^{ \pm}\right)=F^{+}+F^{-}, \quad F^{ \pm}=\frac{1}{2 \varepsilon^{2}} \exp \left\{-\frac{\left(\beta^{ \pm}\right)^{2}}{4 \varepsilon^{2}}\right\}, \\
\cos \beta^{ \pm}=\frac{16-\left[(r \pm)^{2}-(\rho \pm)^{2}\right]^{2}+16 r^{ \pm} \rho^{ \pm}}{16+\left[\left(r^{ \pm}\right)^{2}-\left(\rho^{ \pm}\right)^{2}\right]^{2}+8\left[\left(r^{ \pm}\right)^{2}-\left(\rho^{ \pm}\right)^{2}\right]}
\end{gathered}
$$

with $r^{ \pm}, \rho^{ \pm}$(18).

We get for $P F\{0001\}$ (see Figure $3 b$ ) $\vec{h}=\{0,0\}$ from formula (19)

$$
P_{\{0001\}}\left(r^{ \pm}, \rho^{ \pm}\right)=F^{+}+F^{-} \text {, }
$$

where

$$
r_{ \pm}=\frac{2 \sin \chi}{|1 \mp \cos \chi|}, \quad \rho=0
$$

thus

$$
\begin{gathered}
P_{\{0001\}}\left(r^{ \pm}\right)=\frac{1}{2 \varepsilon^{2}}\left\{\exp \left\{-\frac{\left(\beta^{+}\right)^{2}}{4 \varepsilon^{2}}\right\}+\exp \left\{-\frac{\left(\beta^{-}\right)^{2}}{4 \varepsilon^{2}}\right\}\right\}, \\
\cos \beta^{ \pm}=\frac{4-\left(r^{ \pm}\right)^{2}}{4+\left(r^{ \pm}\right)^{2}} .
\end{gathered}
$$

In Figures $3 a$ and $3 b P F\{10 \overline{1} 0\}$ and $\{0001\}$ are shown recpectively for beryllium (continuous line) and for the model function (dotted line). 


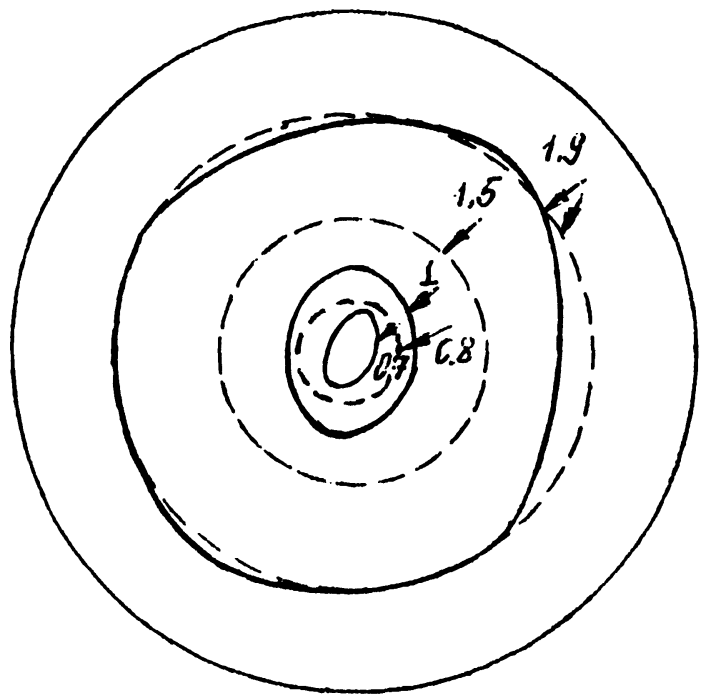

Figure 3a $P F\{10 \overline{10}\}$ for beryllium (continuous line), model function (dotted line).

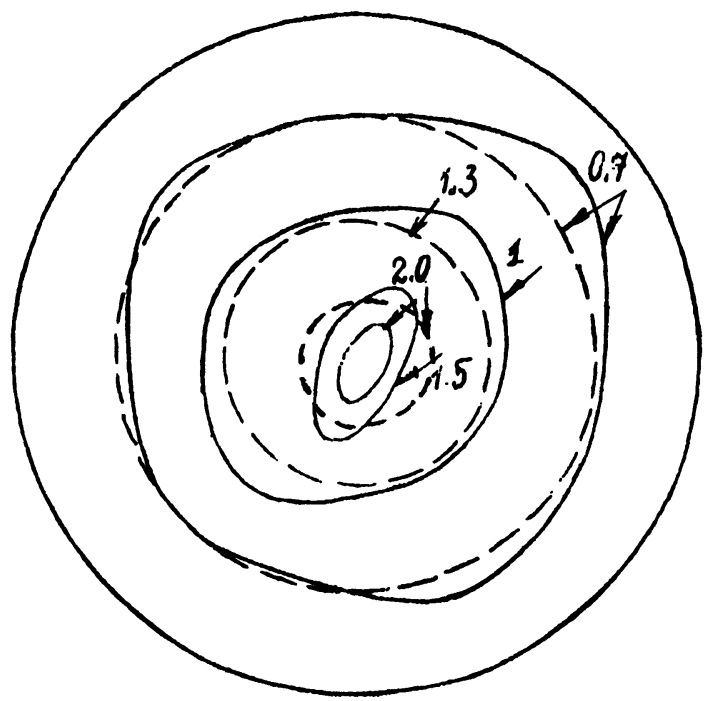

Figure 3b $P F\{0002\}$ for beryllium (continuous line), model function (dotted line). 


\section{CONCLUSION}

In the present paper we get ultrahyperbolic differential equations that allow us to calculate the domains of dependence for pole figures with different lattice symmetries. We get the methods of continuation of solutions of ultrahyperbolic equations and domains of dependence of pole figures. Using these results we can calculate the domains of $P F$ without measuring.

\section{Acknowledgements}

The author thanks T.M. Ivanova for calculation the formula (3).

The auther wishes to thank the reviewers for valuable comments on the manuscript.

\section{Appendix}

For the domains

$$
G^{ \pm}=\left\{r^{ \pm}+\rho^{ \pm} \geq 2\right\}=\{\sin \chi+\sin \theta \geq|\cos \chi \mp \cos \theta|, \quad 0 \leq \chi, \quad \theta \leq \pi,
$$

we get

$$
G^{ \pm}=\left\{|\chi-\theta| \leq \frac{\pi}{2}\right\}, \quad G^{-}=\left\{\frac{\pi}{2} \leq \chi+\theta \leq \frac{3 \pi}{2}\right\} .
$$

Thus for the domain $G=G^{+} \cap G^{-}$we have

$$
G=\left\{\frac{\pi}{2}-\theta<\chi<\frac{\pi}{2}+\theta\right\}
$$

In picture 4 the domains $G^{+}, G^{-}, G$ are shown. In Figures $4 a-4 c$ the domains $G^{+}$, $G^{-}, G$ are shown, where

$$
G^{ \pm}=\left\{2 \leq r^{ \pm}+\rho^{ \pm} \leq a\right\}=\left\{1 \leq \frac{\sin \chi+\sin \theta}{\left|\cos \chi \mp \cos \theta_{i}\right|} \leq \frac{a}{2}\right\}, \quad G=G^{+} \cap G^{-},
$$

for cases:

in Figure $4 a$ we have $2<a<2(2+\sqrt{3})$, or $0<\theta^{*} \leq \frac{\pi}{6}$, where

$$
\theta^{*}=\tan ^{-1}\left(\frac{a}{2}\right)-\frac{\pi}{4}
$$

in Figure $4 b$ the parameter $a$ is equal to $2(2+\sqrt{3})$ or $\theta^{*}=\frac{\pi}{6}$;

in Figure $4 c$ we have $2(2+\sqrt{3})<a<+\infty$ or $\frac{\pi}{6}<\theta^{*}<\frac{\pi}{2}$.

When $a=\infty$ we get the maximum domain $G$ given in Figure 4 . We can see all the variants, written in $1 a-1 d, 2 a-2 d$ and represented in Figures $1 a-1 d$, $2 a-2 d$ respectively. 
DOMAINS OF DEPENDENCE

197

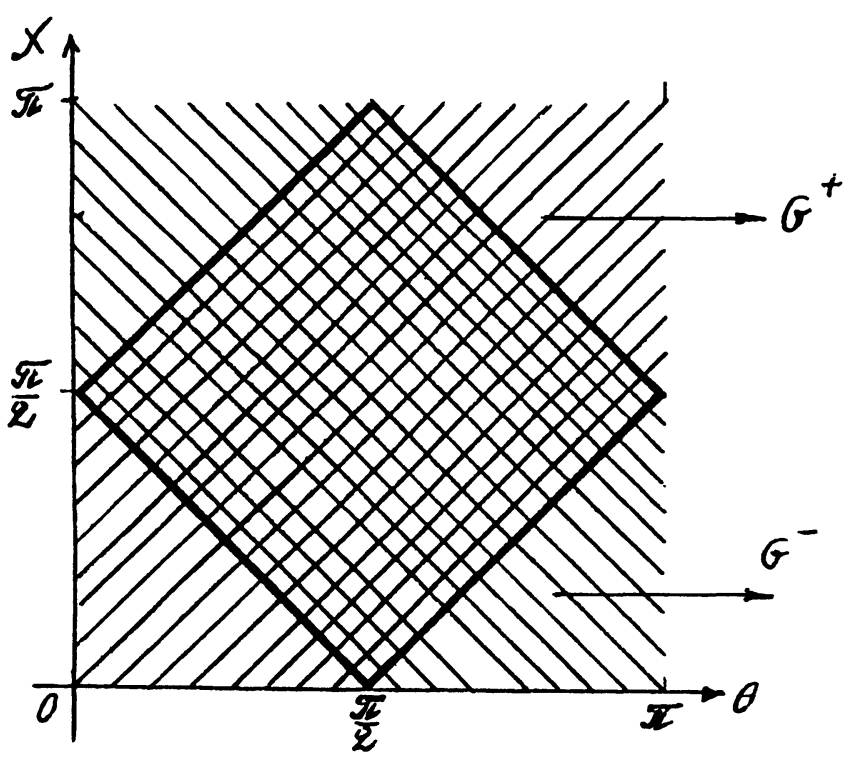

Figure 4 The domain $G$ when $a=\infty$.

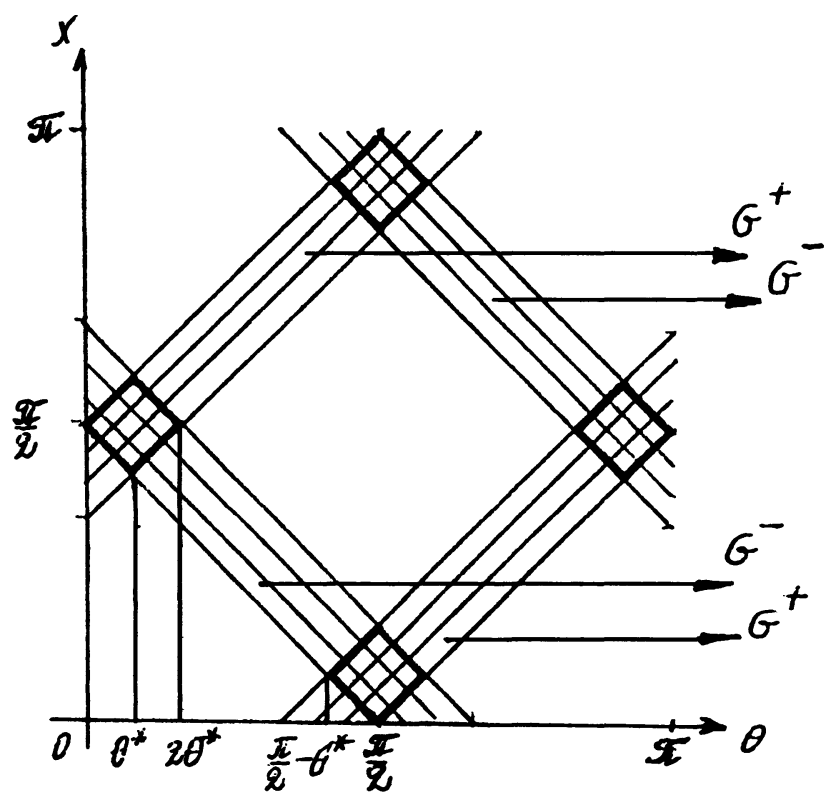

Figure 4a The domain $G$ when $2 \leq a<2(2+\sqrt{3})$. 


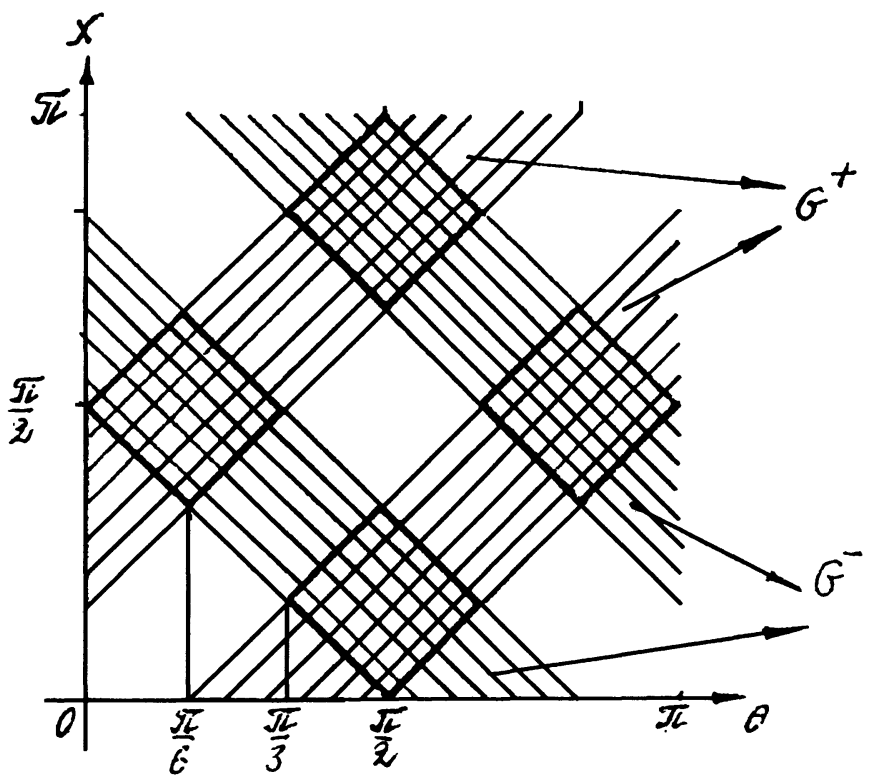

Figure 4b The domain $G$ when $a=2(2+\sqrt{3})$.

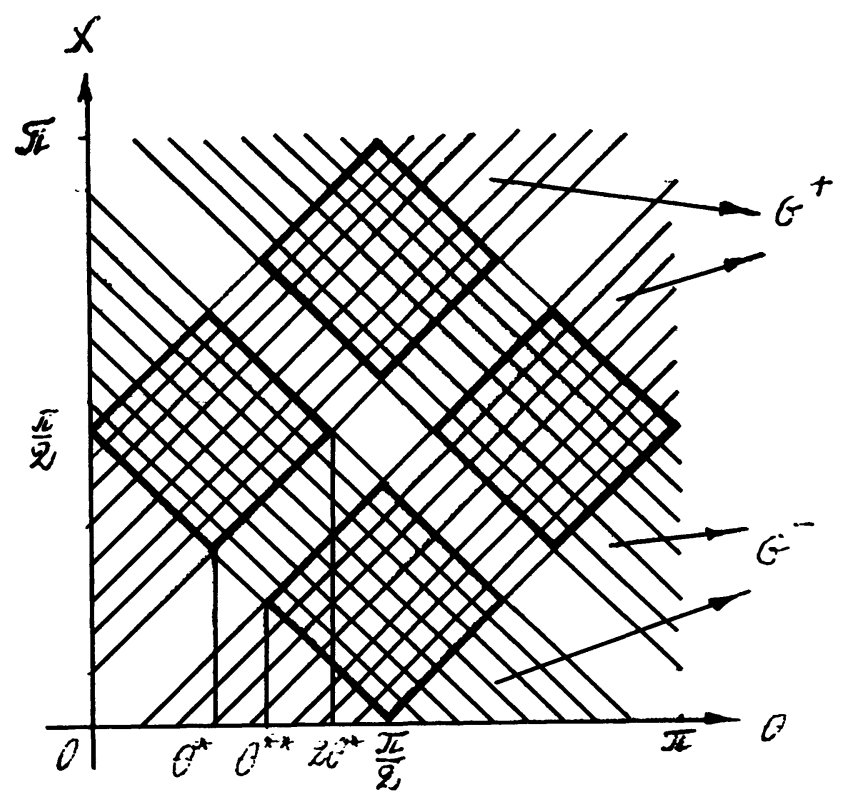

Figure 4c The domain $G$ when $a>2(2+\sqrt{3})$. 


\section{References}

Bunge, H. J. (1982). Texture Analysis in Materials Science. London: Butterworths.

Courant, R. (1962). Partial differential equations. New York, London.

Ivanova, T. M. and Savyolova, T. I. (1993). Calculation of domains of dependence of pole figures for quartz. Physics of Earth, N6, 53-58, (in Russian).

Korn, G. and Korn, T. (1968). Mathematical handbook for scientists and engineers. Mc Graw-Hill Book Company, New York.

Matthies, S. (1979). On the Reproducibility of Orientation Distribution Function of Texture Samples from Pole Figures (Ghost Phenomena). Phys. Stat. Sol. (b), 92(9), K 135-K 137.

Savyolova, T. I. (1982). On solution of inverse problem of diffraction. Reports of Academy of Sciences USSR, 266(3), 590-593, (in Russian).

Savyolova, T. I. (1993). Inverse formulas for orientation distribution function. ICOTOM 10, Tenth International Conference on Textures of Materials, Clausthal, 172.

Savyolova, T. I. (1988). Properties of solving of one problem of diffraction. Izvestiy Vuzov, Mathematics, 4(88), (in Russian).

Vilenkin, N. J. (1965). The special functions and theory of representations of groups. Science, Moscow (in Russian). 